\title{
Medium Buch
}

\section{Wolfenbütteler interdisziplinäre Forschungen}

In Zusammenarbeit mit dem Wolfenbütteler Arbeitskreis für Bibliotheks-, Buch- und Mediengeschichte

herausgegeben von der Herzog August Bibliothek

Redaktion: Hartmut Beyer und Sandra Simon

\section{$1(2019)$}

Praxeologische Studien zur historischen Buchwissenschaft Herausgegeben von Ute Schneider

Harrassowitz Verlag $\cdot$ Wiesbaden 
Anschrift der Redaktion

Herzog August Bibliothek, Postfach 13 64, 38299 Wolfenbüttel

\section{(c) ()}

Dies ist ein Open-Access-Titel, der unter den Bedingungen der CC BY-SA 4.0-Lizenz veröffentlicht wird. Diese erlaubt die kommerzielle Nutzung, Verbreitung und Vervielfältigung in allen Medien. Weitere Informationen: https://creativecommons.org/licenses/by-sa/4.0/deed.de

Die Bedingungen der CC-Lizenz gelten nur für das Originalmaterial. Die Verwendung von Material aus anderen Quellen (gekennzeichnet durch eine Quellenangabe) wie Schaubilder, Abbildungen, Fotos und Textauszüge erfordert ggf. weitere Nutzungsgenehmigungen durch den jeweiligen Rechteinhaber.

Bibliografische Information der Deutschen Nationalbibliothek

Die Deutsche Nationalbibliothek verzeichnet diese Publikation in der Deutschen Nationalbibliografie; detaillierte bibliografische Daten sind im Internet über https://www.dnb.de/ abrufbar.

Informationen zum Verlagsprogramm finden Sie unter

https://www.harrassowitz-verlag.de/

() bei den Beiträger/innen.

Verlegt durch Otto Harrassowitz GmbH \& Co. KG, Wiesbaden 2020

ISSN 2699-9625

eISSN 2748-5161

DOI: $10.13173 / 2699-9625$

ISBN 978-3-447-11304-5

Ebook-ISBN 978-3-447-39178-8

DOI: $10.13173 /$ WIF.1 


\section{Inhalt}

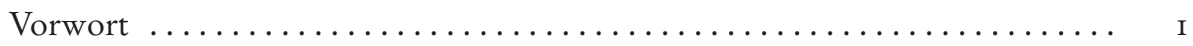

\section{FORSCHUNGSBERICHT}

Charlotte Kempf

Buchwissenschaft und Praxeologie - ein Bericht

\section{BUCHGATTUNGEN UND IHR GEBRAUCH}

Ruth Reicher

Magisches Medium - Buchwürdige Bücher der mittelhochdeutschen

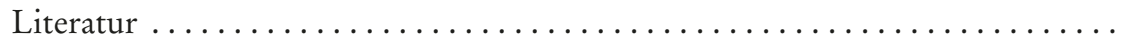

Sebastian Speth

Gedenkbuch und Register. Formen individueller Textaneignung in ,Reineke'-Drucken des I6. Jahrhunderts $\ldots \ldots \ldots \ldots \ldots \ldots \ldots \ldots \ldots \ldots \ldots \ldots$ I

Petra Feuerstein-Herz

Von heimlichkeit der Natur. Benutzungsspuren in alchemischen

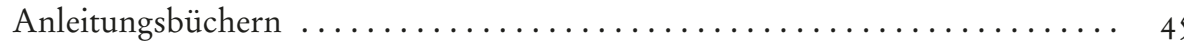

Elisabeth Natour

„Even in this printing age of ours“ - Hippologische Literatur und

soziale Mobilität im Europa des I7. Jahrhunderts ................. 69

Benedikt Brunner

Die gedruckte Leichenpredigt als Erbauungsbuch - eine

Erfolgsgeschichte des 17. Jahrhunderts? .................. 87

Christian Sinn

Scherze in Quart: Zur literarischen Darstellung historischen

Buchgebrauchs bei Jean Paul, Gottfried Keller, Wilhelm Raabe und

Theodor Fontane

Jan Henschen

Das Drehbuch - ein Buch zum Gebrauch? Filmskripte und ihre

Veröffentlichungen als Buch in den I920er Jahren

Na'ama Zussman

Artists' Books - A Critical Field of Experience 


\section{SPUREN DES BUCHGEBRAUCHS}

Maik Schmerbauch

Pfarrbibliotheken als Förderer der Lesekultur im Bistum Hildesheim

von der Mitte des i 9. Jahrhunderts bis zum Ende des Ersten Weltkrieges ..... I 53

Gudrun Wirtz

„Brüderlichkeit und Einheit“: Drucke der slowenischen

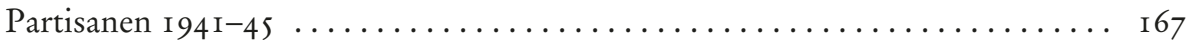

Martin Krickl

Die Bucheinlage - Annäherungen an ein verdecktes Objekt 189

\section{TAGUNGSBERICHT}

Julia Bangert

„Das gebrauchte Buch / The Used Book“ .................... 209

NACHWUCHSFORUM

Julia Schweisthal

Jüdische Pädagogik im „Jahrhundert des Kindes“

\section{PROJEKTBERICHTE}

Elisabeth Engl

Das Projekt OCR-D - Ein Fortschrittsbericht zur

Volltextdigitalisierung frühneuzeitlicher Drucke $\ldots \ldots \ldots \ldots \ldots \ldots \ldots \ldots$

Bettina Gierke

Der Fachinformationsdienst Buch-, Bibliotheks- und

Informationswissenschaft eröffnet neue Möglichkeiten für

die Buchwissenschaft 


\title{
Scherze in Quart \\ Zur literarischen Darstellung historischen Buchgebrauchs bei Jean Paul, Gottfried Keller, Wilhelm Raabe und Theodor Fontane
}

\begin{abstract}
„Bücher haben auch ihr Glücke: Wann sie nicht gesaltzen seyn, / Fasst man dennoch gute Würtze, Pfeffer oder Saffran drein; / Kümmt es dir, ich bin zu friede, liebes Buch, nur auch so gut, / Wann mit dir nur in geheime niemand was verschämtes thut “. ${ }^{\mathrm{I}}$ Mit dem 96. „Desz andren Tausend Siebendes Hundert“ Sinn-Spruch seiner Sinngedichte spinnt Friedrich von Logau eine zuvor schon von ihm begonnene Reflexion über den Buchgebrauch fort, deren nicht selbstverständlicher Kontext von Glück, Gewürzen und Scham auch noch spätere Rezeptionen in sein ästhetisches Spiel zieht. So wie es Gottfried Keller ist, der nicht erst in Das Sinngedicht (I88I), sondern bereits in Die drei gerechten Kammacher (I 856) auf sich über Seiten erstreckenden Sätzen die durch kein Ordnungsprinzip gesammelten Miniaturen einer Lebenswelt aufzählt, zu der neben Gewürzen und anderen Dingen eben auch Bücher, freilich ebenso ganz unterschiedlicher Art, gehören:

[...] ein Körbchen, aus wohlriechenden Halmen geflochten, so wie eines, aus Glasperlen und Gewürznägelein zusammengesetzt; endlich ein kleines Buch, in himmelblaues geripptes Papier gebunden mit silbernem Schnitt, betitelt: Goldene Lebensregeln für die Jungfrau als Braut, Gattin und Mutter; und ein Traumbüchlein, ein Briefsteller, fünf oder sechs Liebesbriefe und ein Schnepper zum Aderlassen [...]. ${ }^{2}$
\end{abstract}

Bücher werden hier nicht nur mit alläglichen Dingen wie in den Enzyklopädien der topica universalis ${ }^{3}$ in eine Ordnung des Wissens eingefügt und so ihrer medialen wie historischen Kontingenz beraubt. Die memoria als Buchprinzip verwandelt vielmehr diese Dinge in ein Andachtsbild, das sich in einem Kirschkern „in welchen das Leiden Christi geschnitten war" ${ }^{4}$ selbst reflektiert und auf den Kontext jener frühneuzeitlichen Erbauungsbücher zurückverweist, die nicht nur rezeptionsgeschichtlich höchste

I Gustav Eitner (Hrsg.): Friedrichs von Logau sämmtliche Sinngedichte. Tübingen I 872 (Bibliothek des literarischen Vereins in Stuttgart; Bd. CXIII), S. 36r.

2 Gottfried Keller: Die Leute von Seldwyla. Hrsg. von Thomas Böning. 2. Aufl. Frankfurt am Main 201 8, S. 207.

3 Wilhelm Schmidt-Biggemann: Topica universalis. Eine Modellgeschichte humanistischer und barocker Wissenschaft. Hamburg 1983.

4 Gottfried Keller: Kammacher. In: Ders.: Leute (wie Anm. 2), S. 207. 
Aufmerksamkeit verdienen, sondern die, wie im Folgenden noch zu zeigen sein wird, Kontingenzen beobachtbar und erfahrbar machten. ${ }^{5}$

Ausgehend von von Graevenitz’ Beobachtung, dass viele Zeitschriften des Realismus sich als ,Gedächtnisbücher für Kultur und Bildung verstanden und präsentiert haben, ${ }^{6}$ vermutet der Beitrag keine reine Fortschrittsgeschichte kultureller Kommunikation. Er fragt vielmehr nach den möglichen Verlusten, indem er eine Blicköffnung durch den Hinweis versucht, dass der memoriale Schrift- und Bildraum, wie er die frühneuzeitlichen, emblematisch verfassten Erbauungsbücher bestimmt, nicht schon darum vergessen werden sollte, weil er eben eine religiöse und damit nach sozialgeschichtlicher Auffassung verdächtige Ideologie ist. Denn es könnte ja auch so sein, dass die bildtheoretische Reflexionsleistung von Andachtsbildern wie z. B. Jörg Ratgebs Auferstebung Christi den Rahmen noch gegenwärtiger Bildauffassungen durch einen iconoclash ${ }^{7}$ sprengt und auch durch Texte reinszeniert wird, die für gewöhnlich als Zeugnisse des Realismus gelten.

Der Reflexion des Buchgebrauchs in Zeitschriften und Büchern des Realismus kommt daher eine potenziell spezifische Erkenntnisleistung zu: So wie die christliche Tradition Bilder zerstörter Bilder und aufgebrochener Rahmen erzeugte, ließe sich an Büchern über zerstörte und missbrauchte Bücher eine Aufklärung von Rezeption ablesen, die man dieser Tradition nicht notwendigerweise zuschreiben wird, auch wenn ihr im ersten Gebot wie in der Figur des Gekreuzigten die Durchstreichung von Bildern und Bilderwartungen eingeschrieben ist.

So reflektiert bereits der zweite Satz von Die drei gerechten Kammacher „jene blutlose Gerechtigkeit, welche aus dem Vaterunser die Bitte gestrichen hat: Und vergib uns unsere Schulden, wie auch wir vergeben unsern Schuldnern! ${ }^{8}$ und markiert damit die Tatsache, dass die bildlose Tradition des Protestantismus und hier v. a. ihr bilderstürmerischer Impuls aus der refomierten Schweiz ihre eigenen Probleme erzeugte, eben die Gerechtigkeit als Selbstgerechtigkeit. Es ist auch hier wieder der Buchgebrauch, der als textimmanente Rezeptionsreflexion aufklärend wirkt: Während Jobst bisweilen aus Langeweile in „einer zerrissenen französischen Sprachlehre für das Volk“ blättert, „war bei dem Bayer ein gut gebundenes Büchlein zu finden, betitelt: ,Die kalte und warme Küpe, ein unentbehrliches Handbuch für Blaufärber‘. Darin war aber mit Bleistift geschrieben: ,Unterfand für die 3 Kreizer, welche ich dem Nassauer geborgt. “ 9

5 Erich Kleinschmidt: Actio per distans. Begriffsstrategien der Sichtbarkeit. In: Maria-Theresia Leuker (Hrsg.): Die sichtbare Welt. Visualität in der niederländischen Literatur und Kunst des I7. Jahrhunderts. Münster 20I 2, S. I9-36.

6 Gerhart von Graevenitz: Memoria und Realismus. Erzählende Literatur in der ,Bildungspresse des deutschen Realismus. In: Anselm Haverkamp, Renate Lachmann (Hrsgg.): Memoria. Vergessen und Erinnern. München 1993 (Poetik und Hermeneutik; XV), S. 283-304.

7 Vgl. Bruno Latour: Iconoclash oder Gibt es eine Welt jenseits des Bilderkrieges? Aus dem Engl. v. Gustav Rossler. Berlin 2002.

8 Keller: Kammacher. In: Ders.: Leute (wie Anm. 2), S. I95.

9 Ebd., S. 204. 
Das zerstörte wie das missbrauchte Buch opponieren in Die drei gerechten Kammacher der Praxis eines jungen Buchbindergesellen, der nicht nur alle Bücher, die er einbindet, auch liest, sondern der die Züns im Unterschied zu den drei Kammachern wirklich liebt. In syntaktischer Analogie zu der oben zitierten Aufzählung der Miniaturen in Züns Lade wird „ein grosser chinesischer Tempel aus Papparbeit mit unzähligen Behältern und geheimen Fächern, den man in vielen Stücken auseinander nehmen konnte "I0 sorgfältig beschrieben, um dann nach wiederum einer Seite mit der Benennung eines doppelten Bodens zu enden, der den innersten Grund des Tempels bildet und in den der Buchbindergeselle einen Liebesbrief legt ,in so hübschen und unbefangenen Worten, wie sie nur das wahre Gefühl findet, welches sich in eine Vexiergasse verrannt hat" ${ }^{\text {"I }}{ }^{\text {I }}$

Die höhere Gerechtigkeit, bei Keller freilich ,Schicksal‘ und nicht ,Gott` genannt, besteht nun darin, dass die selbstgerechte Züns nie von dem allerschönsten Brief, der je geschrieben wurde, erfahren wird. Das ist erstens intratextuell durch eine nachvollziehbare Notwendigkeit des Erzählens und der damit verbundenen Trennung in Erzähler- und Figurenrede gut begründet: Würden Figuren wie die Züns in dieselben Reflexionen und Beschreibungen verfallen wie der anonyme Er-Erzähler, so käme es innerhalb der erzählten Zeit kaum zu Kommunikationen zwischen ihnen. Aber auch extratextuell gelingt Kommunikation bereits im Alltag nur dadurch, dass Kontingenzen, wie die oben vom Erzähler interpretierte Korrelation von Lade und Tempel als Kunstkammer voller Miniaturen, ausgeblendet werden. Deshalb gilt zweitens, dass die Leserinnen und Leser der Novelle nicht weiterlesen könnten, wenn sie sich in ihrer Imagination in eine gleichsam post-erbauliche Meditation dieser Kunstkammern verlören. Metatextuell gesehen aber gilt umgekehrt, dass nicht nur bei Keller materiell singuläre Medien mit hoher Darstellungsdichte und scheinbarer Kontingenz die symbolisch generalisierten Kommunikationsmedien ${ }^{\mathrm{I} 2}$, vor allem Macht, Geld, Liebe, Kunst und Wahrheit, im Medium der Kunst als wahre Kontingenz reflektieren. ${ }^{\text {I3 }}$

Der deutschsprachige Realismus entzieht symbolisch generalisierten Kommunikationsmedien wortwörtlich den Kredit, indem er an die bildtheoretische Reflexionsleistung früherer Medien erinnert. ${ }^{I 4}$ Denn die Selbstinterpretation späterer Kommunikationsmedien als soziale Endgültigkeit gilt nur solange, wie sie sich selbst als Medien zum

Io Ebd., S. 2 I 2.

I I Ebd., S. 2 I 3.

I 2 Niklas Luhmann: Die Gesellschaft der Gesellschaft. Frankfurt am Main 1997, v. a. S. 320-378.

I 3 Luhmann spielt in Die Kunst der Gesellschaft (1995) bereits durch die Ambivalenz des zwischen genetious subjectious und genetious objectivus schwebenden Titels auf die Sonderrolle der Kunst an: Sie ist zwar auch nur Teilsystem der modernen Gesellschaft. Aber wenn nach Luhmann alles, was existiert, nur dadurch existiert, dass darüber kommuniziert wird und Kunst, v. a. die Literatur, als Reflexion nicht nur über diese Tatsache, sondern über die konkrete Form solcher Kommunikation verstanden werden kann, belegt dies ihren reflexiven Mehrwert gegenüber anderen Teilsystemen wie etwa die Wirtschaft.

I 4 Vgl. meinen Beitrag Konversion als Darstellungsdenken. Zur bildtheoretischen Reflexionsleistung literarischer Texte am Beispiel von Brentano, Kingsley und Broch. In: Winfried Eckel, Nikolaus Wegmann (Hrsgg.): Figuren der Konversion. Paderborn 20I4, S. 56-8 I. 
Verschwinden bringen. Ihre Selektions- und Kombinationsmechanismen werden jedoch wie z.B. die Lade der Züns als Serien separater narrativer Vignetten explizit reflektiert. Der Bezug zur Emblematik besteht darin, dass innerhalb der sogenannten gelehrten Emblematik wie bei Hermann Hugo oder Jacob Cats Bilder innerhalb der picturae, v.a. in ihren Ecken und Rändern Bildlichkeit reflektierten, d.h. eine Art eigene Bildlogik etablierten. Aber auch das Medium Buch geriet innerhalb der picturae selbst und nicht nur durch die intertextuellen Referenzen der subscriptiones in den Blick. Dabei ist vielleicht folgende Unterscheidung hilfreich: Werden Bücher in den Emblembüchern in visueller oder narrativer Form lediglich aufgezählt, v. a. unter der Perspektive missbrauchter, zerstörter oder vergessener Texte, kann von einem literalen Buchgebrauch gesprochen werden, während der zentrale Wechsel vom Kunstbuch zum Erbauungsbuch ${ }^{15}$ einen strukturellen Buchgebrauch kennzeichnet, indem nicht nur Bücher, sondern auch alle naturalen oder skripturalen Zeichen dem Zweck der ,Erbauung durch das Buch` untergeordnet werden. Dies schliesst die oben skizzierten internen Medienreflexionen jedoch nicht grundsätzlich aus.

Die nachfolgenden Bemerkungen beschränken sich daher auch nicht auf das thematisch naheliegende, schon lange etablierte Theorem gegenwärtiger Literaturwissenschaft: die Materialität der Kommunikation anhand der Medialität des Buches. ${ }^{16}$ Sie unternehmen etwas undisziplinierte Ausflüge in das Reich jener Bücherlustigkeiten deutschsprachiger Literatur, die sich, in ein eigentümliches Kontinuum von Theologie und Satire gespannt, im Kanon der Weltliteratur nicht so recht beheimaten konnten, sieht man von einem Grundtext der hier angesprochenen Tradition ab, dem Narrenschiff Sebastian Brants und dessen Titelbild, dem Büchernarr.

Diese satirische Tradition reflektiert sich und andere im Buchgebrauch als heterodoxe Theologie, - ,Theologie' sei hier als Rede von einem dem Menschen nicht verfügbaren absolutum her, nicht aber konfessionalistisch oder dogmatisch verstanden. Sie wird, so die These des Beitrags, gerade auch von jenen Autoren verwendet, die wie Jean Paul, Gottfried Keller, Wilhelm Raabe und Theodor Fontane auf das freie Spiel in der Darstellung ästhetischer Ideen zielen, „, welches sich von selbst erhält und selbst die Kräfte dazu stärkt“, ${ }^{17}$ nicht zuletzt deshalb, weil sie keine Ästhetizisten sind, sondern für Toleranz kämpfen. Zu diesem funktional verstandenen, nicht um sich selbst kreisenden Spiel, das die Rezeption von physischen wie moralischen Zwängen zu entbinden versucht, gehört nicht zuletzt, dass in ihren Texten Bücher entgegen ihrer ursprünglichen Bestimmung als Ware gebraucht werden: „Der Landkrämer Seirich

Is Ingrid Höpel: Emblem und Sinnbild. Vom Kunstbuch zum Erbauungsbuch. Frankfurt am Main 1987.

I6 Hans Ulrich Gumbrecht, K. Ludwig Pfeiffer (Hrsgg.): Materialität der Kommunikation. Frankfurt am Main i 995 .

I7 Immanuel Kant: Kritik der Urteilskraft. Werkausgabe Bd. X. Hrsg. von Wilhelm Weischedel. Frankfurt am Main. S. 249. (KdU $\mathbb{4} 49)$. 
überraschte uns mit dem größten Bücherschatz, zumal an Novitäten, wovon schon ein Theil geleimt um den Ofen hing, Kaffeesäcke in Quart und Pfefferdüten in Oktav“. ${ }^{18}$

Mendel hingegen, der Herausgeber der Papiere des verstorbenen Gelehrten J. P. F. Hasus, schämt sich

[...] das in deutscher Sprache beschmierte Papier, da es keine anderthalbe Pfund wog, großen Gewürzhändlern anzubieten, deswegen liess ich [Mendel] alles, wie man sieht, genau und ohne Druckfehler abdrucken, damits einige Zentner würde und man es besser einem hiesigen Gewürzhändler antragen könnte: dabei kann mans noch vorher alle deutsche und polnische Gelehrte [...] zu ihrer Lust durchlaufen lassen. ${ }^{19}$

Das Buch vermehrt den Wert der Papiere weniger über die Auflagenhöhe als durch die Tatsache, dass es die Grundlage für die Produktion weiterer Bücher ist. Bücher regen zu einem Nachdenken an, das wiederum zu Papier gebracht werden muss.

Im Falle von Hasus aber ist der Autor bereits vor seinem Tod tot, da der Teufel des Nachts in seinen Körper fährt und diesen als satirische Schreibmaschine missbraucht. Damit wird ein zentraler Topos historischen Buchgebrauchs reflektiert, das Buch Gottes nämlich, in das jeder Mensch seine am Tage begangenen Handlungen einzutragen und von ihnen Rechenschaft abzulegen hat. Der hasenfüßige, sanftmütige Gelehrte Hasus, der in der Nacht zum Werkzeug des Teufels mutiert, um nicht nur scharfsinnige und bittere, sondern auch unzüchtige Texte zu schreiben, stellt jedoch nur den Normalfall der Buchproduktion dar, wie Mendel weiß, weil „Bücherlustige hernach das Buch gar nicht begehren würden, wenn sie sähen, dass es nicht vom Teufel geschrieben worden $[\ldots]^{\text {“20. }}$.

Jean Pauls Reflexionen über den möglichen Buchgebrauch im Buch, von der Auswabl aus des Tenfels Papieren bis hin zur Ankündigung des nachstehenden Pestitzer Realblattes im Komischen Anhang zum Titan, wurden im Kontext Hegelianischer Rezeption immer wieder sehr kritisch aufgenommen. Bei ihnen geht es jedoch nicht um das Erzählen einer Handlung, deren Inhalte sich Philosophemen zuordnen und dann bewerten lassen, sondern um Katachresen, Gedankenbrüche und um Schreibexperimente, die, wie im Falle von J. P. F. Hasus, geradezu scheitern müssen, da durch den modus tollendo tollens die Falschheit von Prämissen aufgedeckt wird. Wer hingegen nur auf die Form solcher Literarisierungen schaut und nicht ihre philosophische Funktion beachtet, wird wie Hegel dem Trugschluss verfallen, solche Texte verdankten sich nicht den Regeln der Logik, sondern seien auf den subjektiven Humor und dessen philosophisch unhaltbaren Kunstbegriff als „Jean Paulsche Trivialität, Abgeschmacktheit der Situationen und Charaktere, wo die Verhältnisse ein ganz Schiefes, leer Gemachtes

I 8 Jean Paul: Sämtliche Werke. Historisch-kritische Ausgabe. Hrsg. von der Preussischen Akademie der Wissenschaften [...]. Abt. I. Bd. 8. Hrsg. v. Eduard Berend. Weimar u. a. I933. Im Folgenden zitiert als HKA Bd., Abt., S., hier: HKA I, 8, S. 268.

I9 HKA I, I, S. 22 I.

20 Ebd., S. 223. 
sind“2I zurückzuführen. Nun zieht aber Jean Paul im Gegenteil mit Beginn seiner satirischen Schreibproduktion und Reflexion historischen Buchgebrauchs die nüchterne Konsequenz, dass die kulturellen und hier vor allem die medialen Vorprogrammierungen einer Kultur ihre Rezeptionen stärker bestimmen als diesen selbst lieb sein kann.

Das gilt v. a. für jenen Aspekt des Buchgebrauchs, der unserer Zeit mittlerweile tatsächlich fremd geworden ist, während er noch im I 8. Jahrhundert kaum vermieden werden kann. Gemeint sind Erbauungsbücher wie Johann Arndts Vom wabren Christentum ( I6 I 3), deren Wirkungsstärke hinsichtlich der allgemeinen Rezeptionshaltung wohl nicht überschätzt werden kann und denen man auch nicht gerecht wird, wenn man sie nur im Sinne einfacher ideologischer Instrumente versteht, die es zu entideologisieren gelte. Vielmehr handelt es sich um memorial hochkomplex konstruierte und emblematisch verfasste Schrift-Räume, die gleichwohl von den historischen Maßen des jeweiligen Schriftträgers abhängig bleiben. Deren Reflexion in literarischen Darstellungen führt dann nicht nur zur Erkenntnis der Materialität von Kommunikation, sondern $\mathrm{zu}$ einem erst noch zu entdeckenden, freilich unerwarteten, wenn nicht unangenehmen theologoumenon. Könnte es doch sein, dass nicht Andacht und Erbauung einmal problematisch waren, sondern dass ihre Profanierung zu politischen Zwecken zu einem, wenn nicht dem zentralen Folgeproblem alteuropäischer Kultur und gegenwärtiger Politik geworden ist. So ,irrational' die metaphysischen Bedürfnisse von Menschen auch sein mögen, man befriedigt sie nicht dadurch, dass man sie philosophisch aufklärt und politisch missbraucht.

Die Verwobenheit beider Aspekte historischen Buchgebrauchs, die Materialität der Kommunikation und die epistemische Relevanz von Erbauung im Kontext von wortwörtlich verstandenen Schreib-Räumen, ist nicht nur wegen ihrer begrifflichen Friktion schwer erkennbar, sondern auch weil es Ende des i 8. Jahrhunderts kaum kulturelle oder gar normierte Vorgaben gibt, wie denn der Raum eines Bogen Papiers zu füllen sei. ${ }^{22}$ Der Schreib-Raum ist gleichsam ein zu weites Feld, in dem dann alles, auch Amalgamierungen von episteme und Erbauung, d. h. nichts mehr möglich ist. Die Formate definieren also leider nicht die Textsorten und diese die Texte, so dass hiervon ausgehend textanalytisch operational vorgegangen werden könnte. Allerdings existieren im Wesentlichen nur drei Papiermaße, deren kognitive Relevanz durchaus reflektiert wird:

Wie die Buchbinder Quart, Oktav, Folio haben, so dünkt mich auch, dass das Band, mit dem der Autor seine Gedanken verbindet, sich nach dem Formate des Buches richten soll, und ein

2I Annemarie Gethmann-Siefert, Bernadette Collenberg-Plotnikov (Hrsgg.): Philosophie der Kunst oder Ästhetik. Nach Hegel. Im Sommer I 826. Mitschrift F. C. H. V.v. Kehler. München 2004 .

22 Jens Löscher: Schreiben. Literarische und wissenschaftliche Innovation bei Lichtenberg, Jean Paul, Goethe. Berlin u. a. 20I4, S. 8I. 
Autor, der eine große Quartseite zu Diensten seiner Feder vor sich siehet, von dem erwartet man starke, lange und weitläuftige Verbindungen der Gedanken. ${ }^{23}$

Das Jean Pauls Faszikel 7 entstammende Zitat reflektiert sich selbst, werden doch Texte, allen voran Berichte, v. a. in juristischen Kontexten zwar zu Faszikeln zusammengebunden, doch stellt sich damit bis heute die Frage nach der internen Kohärenz der einzelnen Berichte. Diese ist nicht notwendigerweise gegeben und daher kann sich auch Jean Paul über die semantische Heterogenität dieser faktualen Textsorte innerhalb seiner fiktionalen Scherze in Quart lustig machen, wenn er aus den vierzig selbst geschriebenen Bibliotheken die Bibliothek von Schriften die Schauessen betreffend dem Leser ganz vorliest.

Um Faszikel als ,Gedächtnisbücher' und diese als ,Geschichte' konstruieren zu können, sind die Paratexte und v. a. die Buchtitel relevant, für die Jean Paul eine eigene Textsorte kreiert, wie er in seinen Vorworten den historischen Buchgebrauch ausgiebig reflektiert. Aber auch das Schreiben wird durch Bücher erlernt. Jean Pauls Fibel schreibt nicht nur als Sekundarschüler eine Fibel für Primarschüler, er konstruiert aus dem Alphabet neue Alphabete und weil er darin Genie zeigt, braucht er selbst keine Fibel. Diese Reflexion auf die Materialität des Buches als notwendiger Erkenntnisbedingung setzt sich in der Jean-Paul-Rezeption Raabes potenziert fort. Indem der Erzähler von Die Chronik der Sperlingsgasse die Zeitung weglegt und zu einem ,alten Buch' greift, überschreitet er mit der medialen zugleich eine kognitive Grenze und betritt strukturanalog zu Träumen innere unermessliche Räume der Stille:

Es war ein einfaches altes Buch, in welches Meister Daniel Chodowiecki gar hübsche Bilder gezeichnet hatte: Asmus omnia sua secum portans, der prächtige Wandsbecker Bote des alten Matthias Claudius, weiland homme de lettres zu Wandsbeck, und recht ein Tag war's, darin zu blättern. ${ }^{24}$

Beide Aspekte, die Reflexion auf die kognitive Relevanz des Buchgebrauchs wie auf dessen komplexe medienhistorischen Bedingungen, führen nun gerade zur Produktion des Buches, das Leserinnen und Leser so in der Hand halten wie der Erzähler der Chronik der Sperlingsgasse den Wandsbecker Boten. Denn während die „Meister in Folio und Quarto, [die] Aldinen und Elzevire“ den Erzähler von den Büchergestellen spöttisch „anglotzen“, ${ }^{25}$ ist es der im Buch beschriebene Buchgebrauch, vom Lesen eines zufällig aufgeschlagenen Bildes im Wandsbecker Boten, das den Erzähler dazu ermutigt, ein „Bilderbuch der Sperlingsgasse; eine Chronik der Sperlingsgasse!“ ${ }^{26} \mathrm{zu}$ schreiben. Obwohl Die Chronik der Sperlingsgasse im Unterschied zum Wandsbecker

23 Jean Paul, Faszikel 7, zweites Heft. „Einfälle“ (keine Paginierung), zit. nach Löscher (wie Anm. 22), S. 8 I.

24 Wilhelm Raabe: Sämtliche Werke. Braunschweiger Ausgabe. Im Auftrag der Braunschweigischen Wissenschaftlichen Gesellschaft nach dem Tode von Karl Hoppe besorgt von Jost Schillemeit. Band I. Bearbeitet Karl Hoppe und Max Carstenn. 2., durchges. Aufl. Göttingen I965, S. I I.

25 Ebd., S. I3.

26 Ebd., S. I3. 
Boten keinerlei Bilder enthält, werden die Ausdrücke „Bilderbuch“ und ,Chronik` zweimal miteinander vertauscht und synonym verwendet. Es wird doch damit, zumal im Kontext der Aldinen und Elzevire, der alte Sinn von Chronik als Weltchronik, also als bebilderte Geschichtsdarstellung, konnotiert. Damit verbunden handelt es sich auch um die „bunten, ewig wechselnden, ewig neuen Bilder dieses großen Bilderbuches, Welt genannt “27, die der langsam erblindende Erzähler narrativ herzustellen weiß, und zwar in jenem Buchformat von Claudius, „in klein $8 .{ }^{\text {“ } 28}$ gedruckt, das im Unterschied zu Folio und Quarto nicht auf selbstbewusste Repräsentation, sondern schlichte Demut setzt.

Es handelt sich um eine nicht nur konfessionell interessante Differenz, die mit den Namen ,Aldus Manutius', ,Louis Elsevir‘ und ,Matthias Claudius' in Die Chronik der Sperlingsgasse verhandelt wird. Denn ,Aldus Manutius‘ steht für den von Erasmus bevorzugten und damit in Konkurrenz zu Luther stehenden humanistischen Typographen im Übergang zum I6. Jahrhundert. Die von Manutius edierte Hypnerotomachia Poliphili setzte durch die emblematische Auslegung von Holzschnitten einen bis heute faszinierenden Text in Szene, der aufgrund seiner Kunstsprache nur von wenigen gelesen wurde und auch deshalb weit mehr als bei Claudius oder gar Raabe einem Bilderbuch ähnelt. Gleichwohl zeichnet diesen Text jene Sprachreflexivität aus, die sich zwar auch bei Jean Paul, Keller und Fontane beobachten lässt, hier jedoch in besonderem Maß auftritt. Die Hauptfigur, der alles, wenn nicht zu viel, liebende, Poliphilius, weiß ein Rätsel zu lösen, das die Inschriften der im Text beschriebenen Bauwerke und die inscriptiones der in den Text eingelagerten emblemata erst lesbar werden lässt: „Et erano exime littere, exacta la sua crassitudine della non aparte et poco più dil diametro dilla quadratura. “29 Durch die Reflexion der materiellen Bedingungen der eigenen Kommunikation, die konkrete geometrische Konstruktion der Schrift durch eine im Verhältnis von knapp ı:ı (Buchstabenbreite:Buchstabenhöhe) gesetzte Type, wird der Text zu einem sich selbst bezeichnenden Ikon, möglicherweise sogar von Manutius selbst, da durch ihn erst diese Schrifttype Eingang in die Buchkultur fand.

Das mit dem Calvinisten Louis Elsevir beginnende Familienunternehmen publizierte hingegen die wesentlichen Wissenschaftstexte von Galileo, Huygens, Descartes u.a. im Übergang zum I7. Jahrhundert. Diese Bücher sind mit Ausnahme allerdings sehr aufwendig gestalteter Titelkupfer zwar weitgehend bilderfrei oder verwenden Bilder nur im Sinne wissenschaftlicher Illustrationen, fallen aber durch die rahmende Funktion reich differenzierter Randornamente gleichsam als Figur des in sich selbst zurückkehrenden Denkens auf.

27 Ebd., S. I4.

28 Matthias Claudius: Asmus omnia secum portans, oder Sämtliche Werke des Wandsbecker Bothen, Erster und Zweyter Theil. Wandsbeck i774, S. V.

29 Francesco Colonna: Hypnerotomachia Poliphili, Vbi Hvmana Omnia Non Nisi Somnivm Esse Docet. Atqve Obiter Plvrima Scitv Saneqvam Digna Commemorat. Venedig I 499, unpaginiert, I.26. Online unter: http://diglib.hab.de/inkunabeln/ I 3-I-eth-2f/start.htm?image $=00038$ [I2.11.2019]. 
Claudius hingegen setzt als Lutheraner im Übergang zum I9. Jahrhundert zwar nur weniges ins Bild, gleichwohl gezielt und im Unterschied zu den „Aldinen und Elzevire" wirkungsorientiert, beginnt doch Der Wandsbecker Bote mit Freund Hain, gleichsam eine ikonische wie ironische Kampfansage an die vorangegangene Bildgenese in Büchern. ${ }^{30}$ Hier sind vor allem die Kupferstiche von Daniel Chodowiecki wesentlich, durch die innerhalb der Ästhetik der Goethezeit eine Grenze gezogen wurde, die Goethe selbst so markierte: „Auch der Künstler wird nie bezahlt, sondern der Handwerker. Chodowiecki der Künstler, den wir bewundern, äße schmale Bissen, aber Chodowiecki der Handwerker, der die elendsten Sudeleien mit seinen Kupfern illuminirt, wird bezahlt. “31

Chodowiecki, der Handwerker, illustriert in populärer Form nicht zuletzt Goethes Werke, er ist aber auch als Künstler der einzige, den sich Georg Christoph Lichtenberg zur Umsetzung seines orbis pictus vorstellen kann und mit dem er sein Leben lang an satirischen und aufklärerischen Projekten wie Der Fortgang des Tugends und des Lasters zusammenarbeitet. Dieser satirischen Bildpraxis verwandt ist trotz des scheinbar erbaulichen Tones bei Claudius die Betonung des Erzählers keinen Roman schreiben, sondern nur Bilder „,in hübsche Rahmen gefaßt, zusammenheften“32 zu wollen. Mit den Rahmen werden die Bilder zwar erst als Bilder wahrnehmbar, doch erst durch Chodowiecki werden auch die Figuren in den ornamentalen Rahmungen (wieder) sichtbar. Wenn Jean Paul bereits 1797 in seiner Erklärung der Holzschnitte die von Chodowiecki-Lichtenberg verwendete Tradition der Bilderklärung parodiert, dann verwundert es nicht, dass Raabe, ein exzessiver Jean-Paul-Leser, diese Parodie durch die Claudius-Rezeption seines Erzählers in Die Chronik der Sperlingsgasse wieder einschleust. Bildinterpretation ist dann einerseits zwar immer durch kontingente Rahmensetzungen bedingt, verfällt aber andererseits gerade nicht dem Verdikt der Beliebigkeit, wenn die Erklärung der Holzschnitte von jedermann als offenkundig falsch erkannt werden kann. ${ }^{33}$ Die Einbildungskraft löst nicht erst bei E. T. A. Hoffmann „mit ihren ungreifbaren ,inneren Bildern' den wirklichen Maler (Chodowiecki, Callot ...) ab. Sie organisiert das Unstrukturierte, indem sie sich selbst imaginäre Skizzen zu möglichen Bildern macht." 34

Muss der geniale Buchillustrator nicht mehr selbst im Buch erscheinen, da er aufgrund seines Wirkens bereits zur Produktion und Rezeption innerer Bilder angeleitet hat, dann kann auch in der beginnenden Moderne die Welt wieder nach dem alten

30 Claudius: Bothen (wie Anm. 28), S. IV.

3 I Johann Wolfgang von Goethe: Leben und Werk. Mit der Biographie „Johann Wolfgang von Goethe" von Anja Höfer und dem Figurenlexikon „Who's who bei Goethe“ von Michael Lösch. (Digitale Bibliothek, Sonderband). Berlin 2006, S. I 5662. [An Johann Friedrich Krafft. Weimar, 9. September 1779].

32 Raabe: Chronik (wie Anm. 24), S. I 5.

33 Vgl. Monika Schmitz-Emans: Die Literatur, die Bilder und das Unsichtbare. Spielformen literarischer Bildinterpretation vom I 8. bis 20. Jahrhundert. Würzburg I999, S. I 86.

34 Ebd. S. 2 II. 
Modell des orbis pictus, als Ensemble emblematischer Text-Bilder neu gesehen werden. Das bilderlose Bilderbuch der Sperlingsgasse mutiert zum „Traumbuch“35, ist in Träumen doch jene „[n]atürliche Magie der Einbildungskraft" ${ }^{36}$ tätig, die gleichwohl mit dem Geltungsanspruch philosophischen Humors verbunden ist. Kants Kritik der reinen Vernunft vor der Nase, die Augen aber bei einer Putzmacherin gegenüber, scheint das philosophische Ich des kurzsichtigen Erzählers verzweifeln zu müssen, bis sein Blick auf ein in der Glasscheibe des Fensters befindliches Bläschen fällt: „Zufällig schaute ich hindurch nach meiner kleinen Putzmacherin, und - ich begriff, daß das Universum sich in einem Punkt konzentrieren könne.“37

Bei der Konzentration des Universums in einem Punkt durch den Geist handelt es sich um einen jener Sätze der Philosophie, den der Erzähler zunächst wie Kant lediglich rezeptiv verstand, dessen auch für die systematische Philosophie heikle Pointe aber nun eben darin besteht, dass der menschliche Geist wesentlich durch die natürliche Magie der Einbildungskraft konstituiert ist. Damit gerät nun weniger Kant ins Wanken, als dass Raabe belegt, dass er mit Kant über Kant hinauszugehen fähig ist.

Die „niedliche kleine“ ${ }^{8} 8$ Putzmacherin verweist als „ombra adorata“39 nicht nur auf eine intermediale Konstellation, sondern mit ihr auf E. T. A. Hoffmann, dessen Spalanzani das Putzmacherinnenbild vorprogrammierte. Es ist dessen Tochter Olimpia, die von Nathanel in Der Sandmann vom Fenster gegenüber nur durch ein Fernglas wahrgenommen werden kann, eben jenes optische Gerät, dass sich der Erzähler in Die Chronik der Sperlingsgasse zu seinem Glück im Unterschied zum wahnsinnig werdenden Nathanel nicht leisten kann: „[D]och beßer als in jeder beschreibung siehst du ihn, wenn du den Cagliostro wie er von Chodowiecki in irgend einem in berlinischen Taschenkalender steht anschaust. - So sieht Spalanzani aus. -“.40

Damit schließt sich der bildlogische Kreis: Im Unterschied zum i 8. Jahrhundert bedarf es im I 9. Jahrhundert keiner Veranschaulichung durch äußere Kupferstiche mehr, vielmehr muss man wie ein Chodowiecki sehen können, um Bücher richtig zu gebrauchen, als Skizzen nämlich zu möglichen inneren, aber auch äußeren Bildern. Denn der Humor als dichterische Einbildungskraft zeigt sich bei Raabe als kommunikative Alternanz von Zeichnung und Narration innerhalb des einen Mediums Papier. Wie dem gelehrten Chronisten Wachholder der Karikaturenzeichner Strobel entgegengesetzt

35 Raabe: Chronik (wie Anm. 24), S. I 8.

36 Jean Paul: HKA I, 5 (wie Anm. I 8), S. 85.

37 Raabe: Chronik (wie Anm. 24), S. I8.

38 Ebd.

39 Ebd. Derselbe Ausdruck bei E. T. A. Hoffmann: Fantasiestücke in Callot's Manier. Blätter aus dem Tagebuche eines reisenden Enthusiasten. Mit einer Vorrede von Jean Paul. Zweite, durchgesehene Auflage in zwei Theilen. Erster Theil mit dem Bildniß des Verfassers. Bamberg I 819 , S. 47.

$4 \circ$ E. T. A. Hoffmann: Der Sandmann. Hrsg. v. Anna Busch. Bearb. v. Janin Afken, Anna Busch, Maike Engelmann. In: Anne Baillot (Hrsg.): Briefe und Texte aus dem intellektuellen Berlin um I 800 . Berlin 2015, S. is. Online unter: http://www.berliner-intellektuelle.eu/ manuscript?Sandmann [29.06.2019]. 
wird, begegnen sich Zeichnung und Narration in einem Buch im Buch: „Er [Strobel] behauptete, meinen dicken Freund, den Doktor Wimmer in München, sehr gut zu kennen, und malte wirklich als Wahrzeichen das heitere Gesicht des vortrefflichen Schriftstellers sogleich auf die innere Seite des Deckels eines daliegenden Buches. "4I

Es verwundert daher auch nicht, dass bei Raabe Freundschaft über den Buchgebrauch konstituiert wird: Der Erzähler Wacholder erzählt seine Lebensgeschichte nicht zuletzt über eine ihm von Franz berichtete Lebensgeschichte durch die Differenz zwischen Tacitus und Bertuch und den mit ihnen Verbundenen. Der zwölfjährige Franz, der im Wald bei seinem Oheim gleichsam wild aufwuchs, soll vom strengen Rektor der Stadt und Vater von Marie erzogen werden. Die Tacitus-Lektüre des Rektors evoziert Arbeit, Studium und Weltabgewandtheit, fortgesetzt in den Strobeliana, in denen Strobel in den Annalen des Tacitus nach einem Ideal sucht, das es nicht mehr gibt:

Aber wo ist denn die Puppe? kam mir damit [mit dem Zitat über die Kampfbereitschaft der Germanen Annales II, I9] plötzlich in den Sinn. Ich schleuderte den Tacitus ins Gras, stellte mich auf die Zehen, reckte den Hals aus, so lang als möglich, und schaute hinüber nach dem Teutoburger Walde. ${ }^{42}$

Mit dem Wort ,Puppe` wird eine metatextuelle Metapher nicht nur des Raabeschen Textes benannt, die zwei inkongruente Kontexte verbindet. ${ }^{43}$ Während im direkten Kontext das Hermannsdenkmal im Teutoburger Wald als ,Puppe‘ im Sinne der unförmigen Nachbildung einer hier zudem unförmigen Gestalt verstanden wird, die in paradoxer Funktion als realer Index für eine imaginierte Vergangenheit steht, bezieht sich dieser Ausdruck im literalen Sinn zugleich auch auf die Puppe, die der kleinen Marie als Ersatz für ihre zerbrochene Puppe zu Beginn des Textes versprochen, aber nie gegeben wurde, so als erinnerte sich der Erzähler an seine Pflicht, kohärent zu erzählen. Gerade aber dieses durchbricht der, Realismus 'Raabes, indem sein Erzähler die Bücher im Sinne der Herzensgüte des Wandsbecker Boten für sein Pflegekind, die Tochter der längst verstorbenen Marie missbraucht: „[...] aus den schweinsledernen Folianten lassen sich so hübsche Puppenstuben bauen; schau einmal her, was für ein prächtiges Bett gibt mein Papierkorb“. ${ }^{44}$

Es sind die Kinder, deren Störungen das Erzählen wachhalten und ganz im Sinne der oben angesprochenen lutherischen Theologie sind, - nicht zuletzt deshalb wird viermal hintereinander „Kinderschrieen is ok een Gesangbauksversch“ wiederholt ${ }^{45}$ und setzen Kinder die Narration in Gang. Während, Tacitus‘ für eine Lektüre als Flucht steht, lockt Bertuchs Bilderbuch zum Nutzen und Vergnügen der Jugend Kinder und Jugendliche

4I Raabe: Chronik (wie Anm. 24), S. 24.

42 Ebd., S. I 48.

43 Vgl. meinen Beitrag „Aber wo ist denn die Puppe?“ Realismus und Puppenspiel bei Storm, Fontane und Raabe. In: Dirk Göttsche, Ulf-Michael Schneider (Hrsg.): Signaturen realistischen Erzählens im Werk Wilhelm Raabes. Würzburg 2010, S. 57-82.

44 Raabe: Chronik (wie Anm. 24), S. 56.

45 Ebd., S. I46-I 47. 
über die eigenen medialen Grenzen hinaus in eine Welt, die sich zu entdecken lohnt. Franz lässt in seinem Leiden den Rektor mit seinem Tacitus in Ruhe und findet dafür

[...] neben dem Nähzeuge der Frau Rektorin ein Buch auf der Bank - ein Bilderbuch, welches mich den Wald, das Jägerhaus, den Ohm, den alten Burchhard, mein ganzes Heimweh zuerst vergessen ließ. Es war ein zerlesener und zerblätterter Band des welt- und kinderbekannten Bertuchschen Werts. Welch eine neue Welt ging mir da auf! ${ }^{46}$

Bilderbücher, Traumbücher, Gesangbücher, Choralbücher, Komplimentierbücher, Kochbücher, Tagebücher, Gebetbücher: Die Chronik der Sperlingsgasse reflektiert nicht nur die mediale Vielfalt und ihren Gebrauch. Sie entwickelt sie auch als nicht unproblematische, gleichwohl aber auch nicht zu verdächtigende Genese eines Vaterlandsverständnisses, das sich wesentlich über die Konjunktion von Volk und Buch vollzieht und dem zugleich als Traumbuch die Realität eines Volkes ohne Buch durchaus bewusst ist:

Ihr habt die Gewohnheit, ihr Prediger und Vormünder des Volks, den Wegziehenden einen Bibelvers in das Gesangbuch des Heimatdorfs zu schreiben; schreibt: Vergesse ich dein, Deutschland, großes Vaterland: so werde meiner Rechten vergessen!47

Diese Kontrafaktur zu Psalm I37,5 wird dann problematisch, wenn sie als gleichsam anti-zionistisch verstanden wird. Sie hätte in der Vergangenheit wenigstens eine Hoffnung dort eröffnen können, wenn sich das Volk ohne Buch auf einen Dialog mit dem Volk des Buches würde besonnen haben. Dieser Dialog hat indes, mit sehr wenigen, leuchtenden Ausnahmen wie die Diskussion zwischen Mendelssohn und Hamann nie existiert, denn „dort, wo man Bücher /Verbrennt, verbrennt man am Ende auch Menschen. “ 48

So ist die Frage nach dem Buchgebrauch, und was man Büchern antut, in ihrem Kern alles andere als nur historisch, sondern so prekär, dass sich darauf kaum eine gute Antwort finden wird: Namhafte, liberale Autoren wie Fontane, dessen Romanwelt ohne seinen Lieblingsdichter Heine, gerade auch hinsichtlich des Umgangs mit der deutschen Sprache, schlicht nicht verstanden werden kann, ästhetisieren Klischees, nicht zuletzt antisemitische. Allenfalls eine reflektierte Rezeption wird damit umgehen können, indem sie solche Klischees als Aufklärung der Gesellschaft durch die Literatur begreift. Während man dann solche Aufklärung im Falle Brentanos sicherlich verneinen, im Falle Fontanes jedoch eher bejahen können wird, gilt gleichwohl, dass der Weitertransport solcher Klischees auch durch Fontane erfolgt. Karl Kraus, dem man gewiss Sprachbewusstsein nicht abstreiten wird, stellt den Prüfstein einer Germanistik, aber auch des größeren Teils der Literaturproduktion und Literaturkritik dar, die bis heute einem Literaturbegriff huldigt, der gewiss nicht mit ,Antisemitismus' gleichzusetzen ist, aber in seinem Beharren auf ,Authentizität', Selbstaussage des Autorsubjekts und

46 Ebd., S. 4I.

47 Ebd., S. I67.

48 Heinrich Heine: Sämtliche Schriften. Hrsg. von Klaus Briegleb. Band I. München I969, S. 284 . 
im Kampf gegen jede ,Rhetorik‘ gerade auch von anspruchsvollster linksintellektueller und philosophischer Position aus Stereotypen fortschreibt, die in der Moderne ihre Geltung längst verloren haben sollten: „Nur der verfügt über die Sprache wie ein Instrument, der in Wahrheit nicht in ihr ist. [...] Dem Subjekt aber, das die Sprache wie ein vergriffenes Ding gebraucht, ist sie selber fremd.“49

Unter diesem Aspekt dürfte die hier vorgeschlagene Reihung von Jean Paul, Keller, Raabe, aber auch Fontane keine Gnade vor den Augen Adornos finden, handelt es sich doch um Autoren, die nichtauthentisch sprechen, indem sie Bücher durch Bücher kommentieren lassen. Adornos Kritik an Heine markiert überdies, zudem im gegenwärtig fragilen politischen Kontext bundespräsidialer Toleranz gegen oder für (?) rechts einerseits und der Gleichsetzung jeglicher Kritik an der Regierungspolitik Israels mit ,Antisemitismus“ bis zur Entlassung hochkarätiger Gelehrter hin andererseits, dass der Streit um die Definitionshoheit, was , jüdisch“ ist, eine lange Vorgeschichte hat, die so explosionsgeladen ist, dass das Schweigen darüber gerade nicht besser wäre, sondern in jenem Kontext aufgearbeitet werden muss, den Arno Gruen als Der Fremde in uns betitelte. Hier geht es dann nicht nur um individuelle Aufarbeitung, sondern auch um die kulturpoetische und kulturhistorische Frage, wann und wo Kulturen sich als Kampf selbst verrieten und nicht die ihnen je eigene Aufgabe der Vermittlung erfüllten.

Dieser übergeordnete Aspekt, dass sich die literarische Reflexion historischen Buchgebrauchs immer schon interkulturell und intermedial als Auseinandersetzung mit dem Fremden in und außer uns vollzieht, lässt sich besonders bei Fontane nachweisen. Die textgenetischen Stufen von Handschrift, Zeitschriftenabdruck und Buch sind hier gut belegt. Da die Zeitschriftenhonorare höher als die Buchvergütungen waren, wollte Fontane seine poetischen Texte einerseits als Fortsetzungen gedruckt sehen. Andererseits verstößt die ,Autobiografie‘ Von Zwanzig bis Dreißig aufgrund ihrer zahlreichen anthropologischen Reflexionen durch Anekdoten, Reiseberichte, Tagebuchumarbeitungen und Implementierung anderer Biografien, gegen den autobiografischen Pakt mit dem Leser und zwingt ihn zur Reflexion seines eigenen Buchgebrauchs durch das Buch selbst.

In Der Stechlin wiederum wird ähnlich wie bei der Lade der Züns durch eine Aneinanderreihung singulärer, heterogener Gegenstände der Raum durch die scheinbar ,realistische` Darstellung eines Zimmers, in dem die Freunde des jungen Stechlin, Rex und Czako, untergebracht sind, entgrenzt:

Das Bett in diesem vorderen Zimmer hatte einen kleinen Himmel und daneben eine Etagere, auf deren oberem Brettchen eine Meißner Figur stand, ihr ohnehin kurzes Röckchen lüpfend, während auf dem unteren Brett ein Neues Testament lag, mit Kelch und Kreuz und einem Palmenzweig auf dem Deckel. Czako nahm das Meißner Püppchen und sagte: „Wenn nicht unser Freund Woldemar bei diesem Arrangement seine Hand mit im Spiele gehabt hat, so

49 Theodor W. Adorno: Die Wunde Heine. In: Ders.: Noten zur Literatur. Frankfurt am Main I98 I, S. 98. 
haben wir hier in bezug auf Requisiten ein Ahnungsvermögen, wie’s nicht größer gedacht werden kann. Das Püppchen pourmoi, das Testament pourvous." 50

Diese Unterordnung des Buches, immerhin des Neuen Testamentes, unter die Puppe scheint figural bedingt zu sein, möchte doch Czako im Gegensatz zu Rex` gläubigem Himmelsblick lieber den Frauen unter die Röcke schauen. Bemerkenswert ist freilich, dass auch für die Figuren selbst unklar bleibt, ob sich diese Szene dem Arrangement des jungen Stechlin verdankt, der durch Testament und Püppchen eine Realität für seine Freunde inszeniert, oder ob durch Buch und Puppe figural unabhängige metatextuelle Kommentare des Erzählers auf die als Spiel gedeutete Realität des Stechlin gegeben werden.

Ähnlich ambivalent ist der Buchgebrauch bereits in L'Adultera, der unter diesem Aspekt vielleicht ergiebigste Text Fontanes, ist er doch vollkommen aus geflügelten Worten, d.h. Zitaten aus Büchern konstruiert, die das Selbstverständnis einer sich selbst als gut ansehenden Gesellschaft dokumentiert, das vom Erzähler gleichwohl untergraben wird. Der Buchgebrauch dient hier als Reflexion kognitiver und sozialer Grenzüberschreitungen. Die Konversationszitationen in L'Adultera zeigen, wie die allgemeinen wissenschaftlichen Prinzipien der damaligen Geschichtswissenschaft wie ,Ursache - Wirkung' oder ,Chronologie‘ außer Kraft gesetzt werden, indem sie auf ältere, ,magische` Verständnisse der Frühen Neuzeit zurückgehen. Geschichte wird hier am Beispiel einer Kopie Tizians als typologisch gedachte Grenze zwischen Ur- und Abbild gedacht. Durch einen hohen Aufwand subtiler Kohärenzen zwischen Büchern und Bildern kann L'Adultera im Unterschied zu Effi Briest einem unwahrscheinlichen happy ending gleichwohl Glaubwürdigkeit verschaffen.

Durch die intermedialen Kohärenzbildungen werden ähnlich wie bei Raabes Chronik der Sperlingsgasse Traumräume konstruiert, die in merkwürdigem Kontrast zum schulbuchgängigen Verständnis von ,Realismus' (als Abbildung ,der' Wirklichkeit) stehen. Hier sind nicht nur Paratexte relevant, sondern die ebenfalls nach dem Muster der Frühen Neuzeit vollzogene Verwandlung alltäglicher Dinge in Erinnerungsbilder qua Syntaktisierung:

An dem Schnittpunkte von Kurfürstendamm und Kurfürstenstraße, schräg gegenüber dem „Zoologischen“, befand sich in der Mitte der siebziger Jahre noch eine große, feldeinwärts sich erstreckende Gärtnerei, deren kleines, dreifenstriges, in einem Vorgärtchen um etwa hundert Schritte zurückgelegenes Wohnhaus, trotz aller Kleinheit und Zurückgezogenheit, von der vorübergehenden Straße her sehr wohl erkannt werden konnte. ${ }^{\text {I }}$

In sich überbietenden Hypotaxen überführt der Erzähler Fontanes in Irrungen, Wirrungen die lineare Sukzession topographischer Kreuzungspunkte in ein Memorialbild. Damit stellen sich Fragen nach dem Zusammenhang von Kognition und schreibräumlichem Denken, damit aber auch nach dem Realismusbegriff selbst.

50 Theodor Fontane: Große Brandenburger Ausgabe (GBA). I. Abteilung: Das erzählerische Werk. 2 I Bände. Berlin I997-, hier Bd. XVII, S. 2 I.

s I Ebd., GBA I/Io, S. s. 
So kann Fontanes Syntax einerseits als Widerstand gegen eine sich selbst immer stärker überholenden Medienbeschleunigung verstanden werden, ist doch, wie Ein Sommer in London eindringlich belegt, die Herstellung von Makulatur die eigentliche Realität, der Memorialbilder durch einen Realismus der Dinglichkeit ${ }^{52}$ entgegengesetzt werden. Andererseits lässt sich aber auch beobachten, dass Fontanes Romane Makulatur nicht nur negativ auffassen, sondern durch eine Anekdotologie im Stile Eberhard von Poggenpuhls entschieden aufwerten: „Denn nach meinen Erfahrungen umschließt die sogenannte Makulatur einen ganz bedeutenden Geschichtsfond, mehr als manche Geschichtsbücher".53

Dem wäre nun zwar unter den diesen Beitrag leitenden Erkenntnisinteressen nichts mehr hinzuzufügen, gleichwohl hängt die episteme entgegen der Aristotelischen Metaphysik ${ }^{54}$ oft genug mit der tyche zusammen und dies scheint vor allem für die literarische Darstellung historischen Buchgebrauchs im Sinne Jean Pauls zentral zu sein. In Navid Kermanis Dein Name kann der Erzähler nur weiterschreiben, weil er die durch den Austausch eines Malerbocks entstehende Höhendifferenz seiner Schreibtischplatte zu einem freiwerdenden und nur unter der Platte unterzubringenden Bürocontainer mit dem ersten Band einer Dünndruckausgabe von Jean Pauls Werken wieder in Balance bringt und ein hilfsbereiter Student bestätigt mit Hilfe seiner Wasserwaage, „daß ein gewöhnlicher Bürocontainer ohne Rollen zusammen mit dem ersten Band der Dünndruckausgabe von Jean Paul exakt die Höhe eines Malerbocks hat." "ss

52 Bernhard J. Dotzler: Echte Korrespondenzen. Fontanes Welt-Literatur. In: Ders.: Diskurs und Medium III. Philologische Untersuchungen. Medien und Wissen in literaturgeschichtlichen Beispielen. München 20I I, S. I76.

53 Fontane: GBA I/r6 (wie Anm. 50), S. 50.

54 Met. E 2: 1027 a 20-28.

55 Navid Kermani: Über den Zufall. Jean Paul, Hölderlin und der Roman, den ich schreibe. München 20I2, S. I I-I 2. 